\title{
Video Article \\ Visualizing Lignification Dynamics in Plants with Click Chemistry: Dual Labeling is BLISS!
}

\author{
Clémence Simon ${ }^{1}$, Corentin Spriet ${ }^{1}$, Simon Hawkins ${ }^{1}$, Cedric Lion ${ }^{1}$ \\ ${ }^{1}$ UGSF - Unité de Glycobiologie Structurale et Fonctionnelle, CNRS, UMR 8576, Université de Lille
}

Correspondence to: Cedric Lion at Cedric.Lion@univ-lille1.fr

URL: https://www.jove.com/video/56947

DOI: doi: $10.3791 / 56947$

Keywords: Chemistry, Issue 131, Click chemistry, SPAAC, CuAAC, lignin, lignification, monolignol, plant biology, cell wall, flax

Date Published: $1 / 26 / 2018$

Citation: Simon, C., Spriet, C., Hawkins, S., Lion, C. Visualizing Lignification Dynamics in Plants with Click Chemistry: Dual Labeling is BLISS!. J. Vis. Exp. (131), e56947, doi:10.3791/56947 (2018).

\section{Abstract}

Lignin is one of the most prevalent biopolymers on the planet and a major component of lignocellulosic biomass. This phenolic polymer plays a vital structural and protective role in the development and life of higher plants. Although the intricate mechanisms regulating lignification processes in vivo strongly impact the industrial valorization of many plant-derived products, the scientific community still has a long way to go to decipher them. In a simple three-step workflow, the dual labeling protocol presented herein enables bioimaging studies of actively lignifying zones of plant tissues. The first step consists in the metabolic incorporation of two independent chemical reporters, surrogates of the two native monolignols that give rise to lignin $\mathrm{H}$ - and G-units. After incorporation into growing lignin polymers, each reporter is then specifically labeled with its own fluorescent probe via a sequential combination of bioorthogonal SPAAC/CuAAC click reactions. Combined with lignin autofluorescence, this approach leads to the generation of three-color localization maps of lignin within plant cell walls by confocal fluorescence microscopy and provides precise spatial information on the presence or absence of active lignification machinery at the scale of plant tissues, cells and different cell wall layers.

\section{Video Link}

The video component of this article can be found at https://www.jove.com/video/56947/

\section{Introduction}

Over the past two decades, the chemical reporter strategy has emerged as a powerful two-step methodology to investigate the dynamics and functions of non-genetically encoded biomolecules. ${ }^{1,3}$ In this strategy a synthetic analogue of the biomolecule of interest with a small modulation - the chemical reporter - is first metabolized by the living organism, and then a chemical probe (e.g., a fluorophore for fluorescence confocal microscopy imaging) is covalently linked to the incorporated reporter via bioorthogonal click chemistry. The probe must react rapidly and specifically with the introduced chemical modification while being inert to any biomolecules present in the living system. In many ways, this method overcomes the limitations of common bioconjugation techniques through the use of highly specific click chemistry ligations thereby providing the opportunity to track metabolites or biomacromolecules that were previously inaccessible in living systems ${ }^{4,5,6}$.

Despite the fast-growing popularity of this powerful method in bacterial and animal cells, reports describing its use in plant biology are surprisingly few and far between $7,8,9,10,11,12$. We were particularly interested in applying this strategy in plants to study the formation of lignin, one of the most prevalent biopolymers on the planet and a major component of lignocellulosic biomass. ${ }^{13,14}$ Lignin is a phenolic polymer that plays a vital structural and protective role in the development and life of higher plants.

It is generally composed of three 4-hydroxyphenylpropanoid moieties: $\mathrm{H}$ ( $p$-hydroxyphenyl), $\mathrm{G}$ (guaiacyl) and $\mathrm{S}$ (syringyl) units respectively derived from three 'monolignols' ( $p$-coumaryl, coniferyl and sinapyl alcohols) that are synthesized via the phenylpropanoid pathway in the cytoplasm of the cell (Figure 1). After being exported to the cell wall, monolignols are oxidized to radicals by peroxidases or laccases after which they undergo purely chemical radical coupling reactions to polymerize to lignin polymers, a process termed lignification. ${ }^{15,16}$ Although lignins strongly impact the industrial valorization of many plant-derived products, the scientific community still has a long way to go to decipher the intricate mechanisms regulating lignification. 


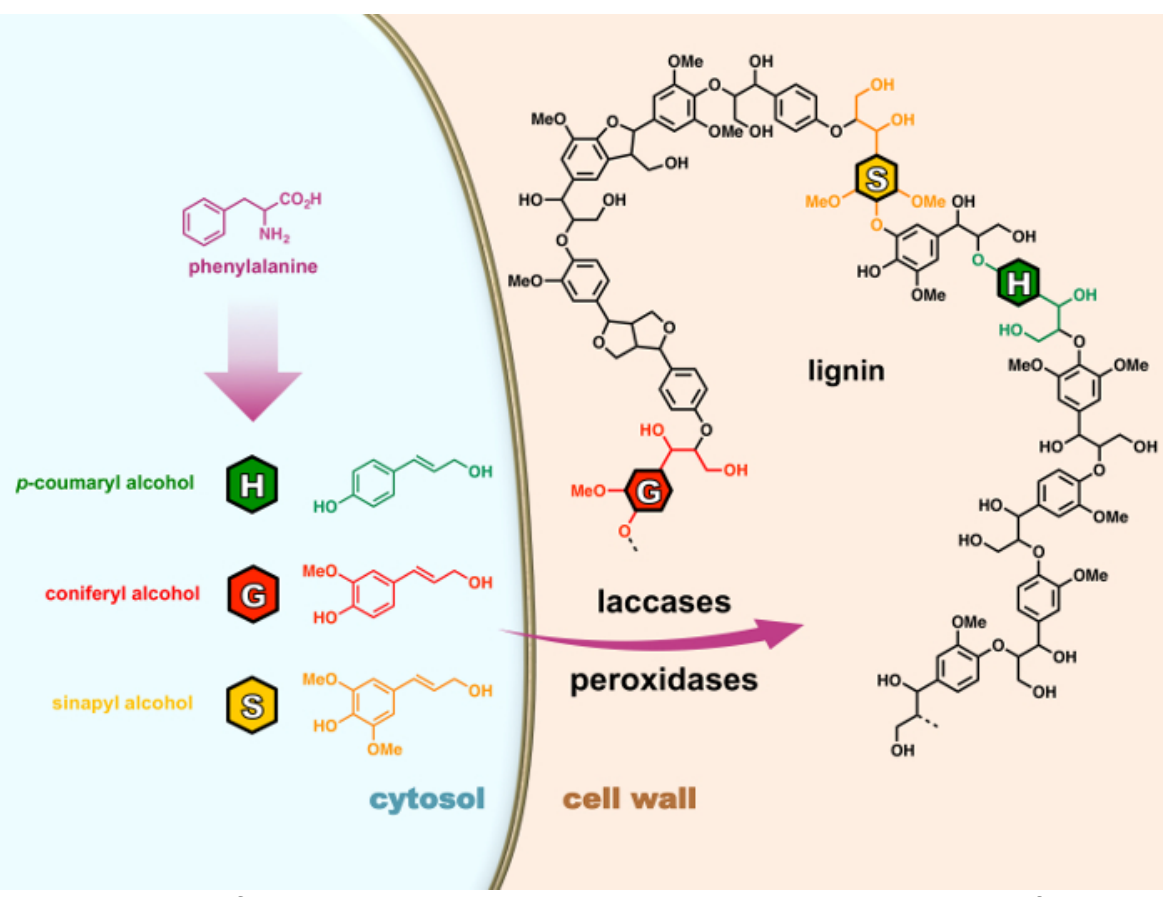

Figure 1: The lignification process in plant cells. Monolignols are biosynthesized from phenylalanine in the cytosol. After being exported to the cell wall, monolignols are oxidized to radicals by peroxidases or laccases after which they undergo purely chemical radical coupling reactions to polymerize to lignin polymers, a process termed lignification. Please click here to view a larger version of this figure.

Although reports on the use of bioorthogonal reactions for glycan analysis are numerous, ${ }^{2,3,17}$ their application examples to other types of biomolecules are fewer. The use of bioorthogonal chemistry for lignin bioimaging purposes was only recently pioneered by Tobimatsu et al. ${ }^{8}$ in Arabidopsis thaliana to provide information about the incorporation of coniferyl alcohol surrogates into the lignin polymer where it forms the $\mathrm{G}$ units, ${ }^{8,9}$ thus demonstrating the proof of concept that chemical reporter strategies are applicable in this context. The use of CuAAC was also illustrated using a different coniferyl alcohol derivative a few month later by Bukowski et al. ${ }^{9}$ However, lignin also contains $\mathrm{H}$ and $\mathrm{S}$ units and a deeper understanding of the lignification process requires more knowledge about how all of the monolignols are incorporated into the polymer and what factors may control its composition. New advances in this field currently depend on the development of efficient methodologies to track multiple chemical reporters simultaneously in living systems. Even though a few articles on glycans have laid the groundwork in recent years $^{18,19,20,21,22}$, dual labeling approaches remain a major challenge in bioorthogonal chemistry. If a reproducible single-labeling click protocol is hard to develop, then dual labeling approaches that require the optimization in tandem of two mutually compatible bioorthogonal reactions on two separate chemical reporters are even harder. The few examples that pioneered this aspect used a combination of strain-promoted azidealkyne cycloaddition (SPAAC) and alkene-tetrazine inverse electronic demand Diels-Alder (DA inv $_{\text {v }}$ reactions to study glycans in animal cells. However, we thought that the bioorthogonality of the DAinv reaction might not be guaranteed in this application owing to the structural features of lignin (which consists of electron-rich substituted cinnamyl-type monomers that can react with electron-poor dienes such as the tetrazine probes used in DAinv reactions) and that this may generate non-specific labeling. In addition, the $\mathrm{DA}_{\text {inv }}$ reaction requires chemical handles that are synthetically difficult to access, as well as being bulky and lipophilic thereby raising the possibility that the rate of incorporation, transport and/or localization of the chemical reporter in vivo may be affected. As we considered that the latter aspect was particularly relevant in the case of a click chemistry approach for studying lignification, we chose a different direction and developed a Bioorthogonal Ligation Imaging Sequential Strategy (BLISS) using a combination of Strain-Promoted Azide-Alkyne Cycloaddition (SPAAC) and Copper Catalysed Azide-Alkyne Cycloaddition (CuAAC) in vivo. ${ }^{23}$

These two reactions are indeed the two main bioorthogonal click reactions that have been used to date, and more particularly in the few examples of lignin imaging that were recently published. ${ }^{8,9}$ Our dual labeling strategy enables the use of an azide moiety on one monolignol reporter and a terminal alkyne on the other, two chemical handles that are i) unreactive towards biologically relevant structures and ii) very small in size (Figure 2). As a result, the impact of these synthetic modifications on the physicochemical properties of the biomolecule under study is minimized thus reducing possible discrepancies between the unnatural and natural monolignol substrates in terms of transport and metabolization rates during the metabolic incorporation step. Although the combination of SPAAC and CuAAC seems very intuitive at first glance, it is to our knowledge only the second example of dual marking using this strategy and the first application on structures other than glycans. 


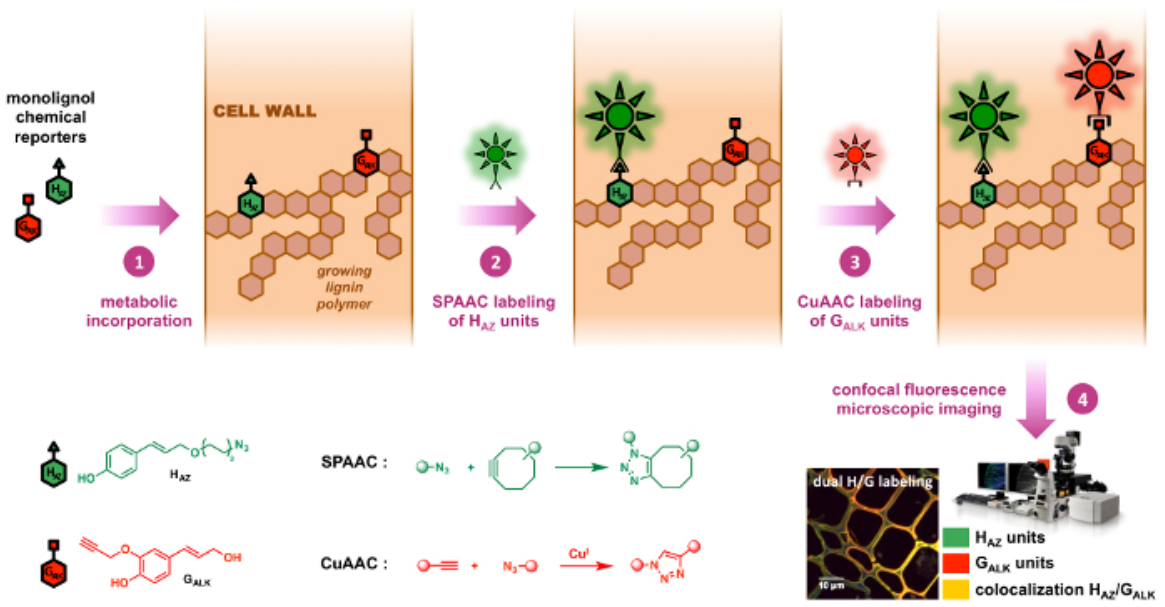

Figure 2: BLISS dual labeling strategy. Chemical reporters $H_{A Z}$ and $G_{A L K}$ are tagged analogs of the native $H$ and $G$ monolignols. They are first incorporated into the growing lignin polymers of the cell walls by exogenous feeding (step 1). Cyclooctyne- and azide-functionalized fluorescent probes are then sequentially ligated to the incorporated reporters by bioorthogonal click chemistry: the SPAAC reaction (step 2) is highly specific of $\mathrm{H}_{A Z}$ units and is followed by a CuAAC reaction (step 3) that is specific of $\mathrm{G}_{\mathrm{ALK}}$ units (step 3), thus allowing the specific localization of both reporters independently in the same sample. Please click here to view a larger version of this figure.

We firstly designed and validated the azide-tagged monolignol reporter $\mathbf{H}_{\mathrm{AZ}}$ (surrogate of $p$-coumaryl alcohol and precursor of lignin $\mathrm{H}$ units), and then devised the BLISS dual labeling strategy in which it is used in tandem with the previously reported alkyne-tagged $\mathbf{G}_{\mathrm{ALK}},{ }^{9}$ (surrogate of coniferyl alcohol and precursor of lignin $\mathrm{G}$ units). In this reproducible protocol developed and tested in flax, an economically important plant species, the dual metabolic incorporation of $\mathbf{H}_{\mathrm{AZ}}$ and $\mathbf{G}_{\mathrm{ALK}}$ into lignin is first achieved prior to sequential SPAAC/CuAAC labeling. Here, tagged $\mathbf{H}_{\mathrm{AZ}}$ units are first specifically labeled via the SPAAC ligation of a cyclooctyne-functionalized fluorophore, followed by CuAAC-mediated ligation of a second fluorescent probe on tagged $\mathbf{G}_{\mathrm{ALK}}$ units. This method was used to investigate the dynamics of lignification processes within plant cell walls and can be applied in vivo to stem cross-sections, living stems as well as seedlings of different plant species.

\section{Protocol}

NOTE: Liquid and solid 1/2 MS media must be prepared beforehand as described in Supplemental Table 1.

\section{Plant Culture}

1. Culture of $\mathbf{2}$ month-old flax plants

1. Sow flax (Linum usitatissimum L.) seeds in plastic pots using potting compost.

2. Grow flax in growth chambers at $22^{\circ} \mathrm{C}$ with a photoperiod of $16 \mathrm{~h} / 8 \mathrm{~h}$ day/night.

3. Equip plants with vertical support after 1 month and grow until they are 2 month-old.

\section{Culture of $\mathbf{2}$ week-old flax seedlings}

1. Wrap 12 flax seeds in a piece of cheese cloth and fix with a rubber band to make a bundle.

NOTE: Carry out the next steps under sterile conditions under a laminar flow fume hood.

2. Place the seed bundle in a previously autoclaved $250 \mathrm{~mL}$ glass bottle.

3. Add $70 \mathrm{~mL}$ of $70 \% \mathrm{EtOH}$ in the bottle and gently stir for $1 \mathrm{~min}$.

4. Carefully remove the EtOH by decantation while leaving the bundle in the bottle.

5. Add $100 \mathrm{~mL}$ of $2 \%$ sodium hypochlorite and stir gently for $10 \mathrm{~min}$.

6. Remove the sodium hypochlorite solution by decantation while leaving the bundle in the bottle.

7. Repeat steps 1.2.5-1.2.6.

8. Add $100 \mathrm{~mL}$ of sterile ultrapure water and stir for $1 \mathrm{~min}$.

9. Repeat step 1.2.8 3 times, on increasing the rinsing time for each rinse (5, 10 and $15 \mathrm{~min})$.

10. Warm solid $1 / 2$ MS medium, pour it in a sterile plant culture box to a height of $2 \mathrm{~cm}$ and let it cool down until solidification.

11. Delicately place each seed on solid medium using sterile forceps.

12. Close the sterile box.

13. Transfer the box to a growth chamber at $22^{\circ} \mathrm{C}$ with a photoperiod of $16 \mathrm{~h} / 8 \mathrm{~h}$ day/night and grow flax seedlings for 2 weeks.

\section{Metabolic Incorporation of Chemical Reporters}

NOTE: Three experimental models are presented below: i) metabolic labeling of stem cross-sections, ii) whole stems and iii) plant seedlings. Each protocol is presented for one biological replicate and quantities can be adapted to the number of required biological replicates. The monolignol solutions are prepared from stock solutions that are made as described in Table 2 prior to the experiment. The stock solutions can be stored several months at $-20^{\circ} \mathrm{C}$. The $\mathbf{H}_{\mathrm{AZ}}: \mathbf{G}_{\mathrm{ALK}}$ or $\mathbf{H}: \mathbf{G}$ proportions can be adjusted to fit all custom-made experimental designs. 
1. Chemical reporter incorporation into 2 month-old flax stem cross-sections

1. Prepare a $300 \mu \mathrm{L}$ chemical reporter solution containing $10 \mu \mathrm{M}$ of $\mathbf{G}_{\mathrm{ALK}}$ and $10 \mu \mathrm{M}$ of $\mathbf{H}_{\mathrm{AZ}}$ in liquid sterile $1 / 2 \mathrm{MS}$ medium.

2. Vortex the $\mathbf{H}_{A Z} / \mathbf{G}_{A L K}$ solution and transfer it to one well of a 48-well plate with a micropipette.

3. Prepare a $300 \mu \mathrm{L}$ negative control solution containing $10 \mu \mathrm{M}$ of $\mathbf{G}$ and $10 \mu \mathrm{M}$ of $\mathbf{H}$ in liquid sterile $1 / 2 \mathrm{MS}$ medium.

4. Vortex the $\mathbf{H} / \mathbf{G}$ solution and transfer it to a second well of the same 48-well plate.

5. Cut the stem of a 2 month-old flax plant at $10 \mathrm{~cm}$ above soil level using a new razor blade.

6. Delicately prepare 50 freehand transversal cross-sections of the stem using the razor blade (approximately $150-250 \mu \mathrm{m}$ thick).

7. Immediately place the cross-sections in liquid sterile $1 / 2 \mathrm{MS}$ medium in a watch glass.

8. Visually inspect and select intact whole cross-sections and randomly distribute 10 of them in the well filled with $\mathbf{H}_{\mathrm{AZ}} / \mathbf{G}_{\mathrm{ALK}}$ solution.

9. Repeat step 2.1.8 for the well filled with $\mathbf{H} / \mathbf{G}$ solution (as negative control).

10. Repeat step 2.1.8 for a third well filled with $300 \mu \mathrm{L}$ of $1 / 2 \mathrm{MS}$ medium (as background control to adjust the fluorescence baseline during the subsequent data processing of confocal microscopy images).

11. Incubate the plate in continuous light in a growth chamber at $20^{\circ} \mathrm{C}$ for $20 \mathrm{~h}$.

12. Remove the monolignol solution in each well with a micropipette.

13. Add $500 \mu \mathrm{L}$ of $1 / 2$ MS medium to each well, stir gently for $10 \mathrm{~min}$ and remove this rinsing solution with a micropipette. Repeat 4 times and proceed to BLISS labeling immediately (section 3).

\section{Chemical reporter incorporation into 2 month-old flax whole stems}

1. Prepare a $5 \mathrm{~mL}$ chemical reporter solution containing $10 \mu \mathrm{M}$ of $\mathbf{G}_{\mathbf{A L K}}$ and $10 \mu \mathrm{M}$ of $\mathbf{H}_{\mathrm{AZ}}$ in liquid sterile $1 / 2 \mathrm{MS}$ medium.

2. Vortex the $\mathbf{H}_{\mathrm{AZ}} / \mathbf{G}_{\mathrm{ALK}}$ solution and transfer it to a 20 -cm-high glass test tube with a pipette.

3. Prepare a $5 \mathrm{~mL}$ negative control solution containing $10 \mu \mathrm{M}$ of $\mathbf{G}$ and $10 \mu \mathrm{M}$ of $\mathbf{H}$ in liquid sterile $1 / 2 \mathrm{MS}$ medium.

4. Vortex the $\mathbf{H} / \mathbf{G}$ solution and transfer it to a $20-\mathrm{cm}$-high glass test tube.

5. Prepare a third glass test tube containing $5 \mathrm{~mL}$ of $1 / 2 \mathrm{MS}$ medium (as background control to adjust the fluorescence baseline during the subsequent data processing of confocal microscopy images)

6. Cut the stem of a 2 month-old flax plant at $10 \mathrm{~cm}$ above soil level using a new razor blade. Discard the root system and lower part of the stem and proceed to the next step with the upper part of the plant stem.

7. Immerse the base of the whole cut stem in the tube containing the $\mathbf{H}_{\mathrm{AZ}} / \mathbf{G}_{\mathrm{ALK}}$ solution. Place the stem in its incubation solution immediately after removal of the root system in order to prevent it from drying.

NOTE: If the method is applied to younger/older plants and/or other species, the volume of monolignol solutions must be adjusted according to the size / age of the plant and the diameter of its stem.

8. Attach the stem to a vertical support to keep it straight.

9. Seal the glass tube with parafilm to avoid evaporation of the solution

10. Repeat steps 2.2.6-2.2.9 for the negative control sample with the $\mathbf{H} / \mathbf{G}$ solution.

11. Repeat steps $2.2 .6-2.2 .9$ for the fluorescence background control sample containing the $1 / 2 \mathrm{MS}$ medium.

12. Incubate each stem for $20 \mathrm{~h}$ in continuous light using a neon light.

13. After $20 \mathrm{~h}$ of metabolic incorporation, remove the stem incubated in the $\mathbf{H}_{\mathrm{AZ}} / \mathbf{G}_{\mathrm{ALK}}$ solution and rinse it with $1 / 2 \mathrm{MS}$ medium.

14. Cut and discard the bottom $1 \mathrm{~cm}$ of the stem using a razor blade and then prepare a $1 \mathrm{~cm}$ high cylinder from the base of the remaining stem.

15. Carefully prepare 20 freehand transverse cross-sections (approximately $150-250 \mu \mathrm{m}$ thick) from this cylinder and place them immediately in a watch glass filled with $1 / 2$ MS medium.

16. Put $500 \mu \mathrm{L}$ of $1 / 2 \mathrm{MS}$ medium in one well of a 48 -well plate.

17. Visually inspect and select intact whole cross-sections and randomly distribute 10 of them in the well filled with $1 / 2$ MS solution.

18. Repeat steps 2.2.13-2.2.17 for the negative control stem incubated with the H/G solution.

19. Repeat steps 2.2.13-2.2.17 for the fluorescence background control stem incubated with $1 / 2 \mathrm{MS}$ medium.

20. Carefully remove the solution in each well with a micropipette.

21. Add $500 \mu \mathrm{L}$ of $1 / 2 \mathrm{MS}$ medium to each well, stir gently for $10 \mathrm{~min}$ and remove this rinsing solution with a micropipette. Repeat 4 times and proceed to BLISS labeling immediately (section 3 ).

3. Chemical reporter incorporation into 2 week-old flax seedlings

NOTE: The following steps are all carried out under sterile conditions.

1. Prepare a $2 \mathrm{~mL}$ chemical reporter solution containing $10 \mu \mathrm{M}$ of $\mathbf{G}_{\mathrm{ALK}}$ and $10 \mu \mathrm{M}$ of $\mathbf{H}_{\mathrm{AZ}}$ in liquid sterile $1 / 2 \mathrm{MS}$ medium.

2. Vortex the $\mathbf{H}_{A Z} / \mathbf{G}_{A L K}$ solution and transfer it to a $20 \mathrm{~cm}$-high glass test tube with a pipette.

3. Prepare a $2 \mathrm{~mL}$ negative control solution containing $10 \mu \mathrm{M}$ of $\mathbf{G}$ and $10 \mu \mathrm{M}$ of $\mathbf{H}$ in liquid sterile $1 / 2 \mathrm{MS}$ medium.

4. Vortex the $\mathbf{H} / \mathbf{G}$ solution and transfer it to a $20 \mathrm{~cm}$-high glass test tube.

5. Prepare a third 20 -cm-high glass test tube containing $2 \mathrm{~mL}$ of $1 / 2 \mathrm{MS}$ medium (as background control to adjust the fluorescence baseline during the subsequent data processing of confocal microscopy images).

6. Delicately remove a 2-week-old flax seedling from the solid $1 / 2 \mathrm{MS}$ medium using a long pair of tweezers (section 1.2. above)

7. Gently transfer the seedling to the tube containing the $\mathbf{H}_{A Z} / \mathbf{G}_{A L K}$ solution, ensuring that its roots are immersed in the solution.

8. Close the glass tube with a plastic cap to avoid evaporation.

9. Repeat steps 2.3.6-2.3.8 for the negative control tube with the $\mathrm{H} / \mathrm{G}$ solution.

10. Repeat steps 2.3.6-2.3.8 for the fluorescence background control tube containing the $1 / 2 \mathrm{MS}$ medium.

11. Incubate each seedling in continuous light in a growth chamber for $20 \mathrm{~h}$ at $20^{\circ} \mathrm{C}$

12. Delicately remove the seedling incubated in the $\mathbf{H}_{A Z} / \mathbf{G}_{A L K}$ solution and rinse it with $1 / 2 \mathrm{MS}$ medium.

13. Delicately prepare 20 freehand cross-sections (approximatively $150-250 \mu \mathrm{m}$ thick) of the hypocotyl.

14. Put $500 \mu \mathrm{L}$ of $1 / 2 \mathrm{MS}$ medium in one well of a 48 -well plate.

15. Visually inspect and select intact whole cross-sections and randomly distribute 10 of them in the well filled with $1 / 2$ MS solution.

16. Repeat steps 2.3.12-2.3.15 for the negative control seedling incubated with the H/G solution.

17. Repeat steps 2.3.12-2.3.15 for the fluorescence background control seedling incubated with $1 / 2 \mathrm{MS}$ medium.

18. Carefully remove the solution in each well with a micropipette. 
19. Add $500 \mu \mathrm{L}$ of $1 / 2 \mathrm{MS}$ medium to each well, stir gently for $10 \mathrm{~min}$ and remove this rinsing solution with a micropipette. Repeat 4 times and proceed to BLISS labeling immediately (section 3).

\title{
3. Dual Fluorescence Labeling of Plant Cross-section Samples by SPAAC and CuAAC
}

\author{
NOTE: The BLISS labeling protocol is identical for all 3 experimental models (section 2).
}

1. Strain-Promoted Azide-Alkyne Cycloaddition (SPAAC) labeling

1. Prepare $1 \mathrm{~mL}$ of a SPAAC solution containing $5 \mu \mathrm{M}$ of DBCO-PEG $4-5 / 6$-carboxyrhodamine 110 in liquid sterile $1 / 2 \mathrm{MS}$ medium. Vortex the solution and keep it in the dark.

2. After the final washing steps of the metabolic incorporation protocol [2.1.13, 2.2.21 or 2.3.19 depending on the chosen experimental design], remove the $1 / 2 \mathrm{MS}$ medium and add $300 \mu \mathrm{L}$ of the SPAAC solution per well with a micropipette.

3. Shield the 48-well plate from light by covering with aluminum foil or by placing it in a box.

4. Gently stir the plate on a mechanical shaker for $1 \mathrm{~h}$ in the dark at ambient temperature.

2. Copper-catalyzed Azide-Alkyne Cycloaddition (CuAAC) labeling

1. Wash each well 4 times as described in step 2.1.13 while keeping the plate in the dark.

2. Prepare $1 \mathrm{~mL}$ of a CuAAC solution containing $2.5 \mathrm{mM}$ sodium ascorbate, $0.5 \mathrm{mM} \mathrm{CuSO}_{4}$ and $5 \mu \mathrm{M}$ of 5 -TAMRA-PEG ${ }_{3}$-azide in liquid sterile $1 / 2$ MS medium. Vortex the solution and keep it in the dark.

NOTE: This CuAAC solution must be freshly prepared prior to use and cannot be stored more than a few days at $4{ }^{\circ} \mathrm{C}$.

3. Remove the $1 / 2 \mathrm{MS}$ medium in each well and directly add $300 \mu \mathrm{L}$ of the CuAAC solution per well using a micropipette.

4. Gently stir the plate on a mechanical shaker for $1 \mathrm{~h}$ in the dark at ambient temperature.

5. Remove the CuAAC solution using a micropipette.

6. Using a micropipette, add $500 \mu \mathrm{L}$ of $1 / 2 \mathrm{MS}$, stir in the dark for $10 \mathrm{~min}$ then remove this washing solution. Repeat twice.

7. Add $500 \mu \mathrm{L}$ of a $7: 3 \mathrm{MeOH} /$ water solution, stir in the dark for $1 \mathrm{~h}$ then remove the solution

CAUTION: Methanol is toxic by skin contact or inhalation, and is designated as a human carcinogen. Its utilization requires chemical fume hoods and gloves.

8. Add $500 \mu \mathrm{L}$ of $1 / 2 \mathrm{MS}$, stir in the dark for 10 min then remove the solution. Repeat 4 times.

\section{Mounting of Samples on Microscope Slides}

1. Place 5 drops of mounting medium on a glass microscope slide.

2. Carefully deposit each $\mathbf{H}_{\mathrm{AZ}} / \mathbf{G}_{\mathrm{ALK}}$ tagged cross-section on the slide.

3. Apply nail polish on two opposite sides of the slide.

4. Cover the slide with a coverslip. Be careful to avoid air bubbles.

5. Remove excess mounting medium using a paper towel.

6. Seal the sample with nail polish on all 4 sides. Let the nail varnish dry at room temperature (approximately $30 \mathrm{~min}$ ).

7. Repeat steps 4.1-4.6 for the negative control $\mathbf{H} / \mathbf{G}$ cross-sections.

8. Repeat steps 4.1-4.6 for the fluorescence background control cross-sections.

9. Store the slides at $4{ }^{\circ} \mathrm{C}$ in the dark until observation under a confocal microscope. NOTE: Prepared slides can be stored for several weeks to several months depending on the quality of the preparation. If a new observation is to be made after storage, ensure that the samples have not dried up.

\section{Image Acquisition by Fluorescence Confocal Microscopy}

1. Turn on all necessary units of the confocal microscopy system in the right order according to the manufacturer's instructions.

2. Select the desired objective with maximal numerical aperture and adapted excitation and emission wavelengths (e.g., obj. 60x, N.A. 1.2. Autofluorescence channel: $\lambda_{\mathrm{ex}} 405 \mathrm{~nm}, \lambda_{\mathrm{em}} 450 \mathrm{~nm} / 25$; SPAAC $\mathrm{H}_{\mathrm{AZ}}$ channel: $\lambda_{\mathrm{ex}} 488 \mathrm{~nm}, \lambda_{\mathrm{em}} 525 \mathrm{~nm} / 25 ;$ CuAAC $\mathrm{G}_{\mathrm{ALK}}$ channel: $\lambda_{\mathrm{ex}} 561 \mathrm{~nm}$, $\lambda_{\mathrm{em}} 595 \mathrm{~nm} / 25$; sequential mode).

3. Select the desired parameters for generation of images in the software (12- or 16-bit required, pixel size adapted to the Nyquist criterion).

4. Place the $\mathbf{H}_{\mathrm{AZ}} / \mathbf{G}_{\mathrm{ALK}}$ slide on the microscope stage and position the sample under the objective lens.

5. Observe and find the desired region of the plant tissue.

6. Acquire high quality confocal images of doubly labeled $H_{A Z} / G_{A L K}$ sample.

1. Select the most fluorescent plane in the Z-axis.

2. Adjust gain, offset and laser power to achieve optimal signal distribution along the whole grey level range for each channel (autofluorescence, $\mathbf{H}_{\mathrm{AZ}}$ and $\mathbf{G}_{\mathrm{ALK}}$ ). The same parameters must be applied for all following acquisitions.

3. Launch the acquisition of the selected image and save the file. Repeat for each relevant area of the sample. NOTE: 3D Z-stack reconstructions may also be acquired if necessary.

7. Using the same settings, acquire images of untagged sample only incubated in $1 / 2$ MS to determine the autofluorescence level (fluorescence background control as described in section 2).

8. Using the same settings, acquire images for the negative control samples incubated with native monolignols H/G to determine non-specific fluorophore adhesion to the sample.

9. Apply data treatment to the acquired images to substract background autofluorescence signals in both $\mathbf{H} / \mathbf{G}$ negative control images and $\mathbf{H}_{\mathrm{AZ}} \mathbf{I}$ $\mathbf{G}_{\mathrm{ALK}}$ images. 
Representative Results

By using the presented BLISS protocol involving synthetic monolignol analogs and bioorthogonal click chemistry, it is possible to visualize the dynamics of the lignification process in living plants. Unlike established techniques for lignin visualization (such as histochemical staining, immunolocalization or autofluorescence), this 'double click' protocol exclusively targets the lignin produced de novo during the metabolic incorporation step and differentiates it from the preexisting lignin of the sample with triple-channel cellular imaging by confocal fluorescence microscopy (Figure 3). $\mathbf{H}_{\mathrm{AZ}}$-units are detected using the excitation and emission wavelengths of the DBCO-PEG $-5 / 6-$ carboxyrhodamine 110 that is specifically clicked onto azide functions of incorporated $\mathbf{H}_{\mathrm{AZ}}$ molecules during the SPAAC step ( $\lambda$ ex $501 \mathrm{~nm} / \lambda_{\mathrm{em}} 526 \mathrm{~nm}$, green channel), whereas $\mathbf{G}_{\mathrm{ALK}}$-units are similarly detected at the characteristic wavelengths of the azidefluor 545 probe that is specifically clicked onto the incorporated terminal alkyne tags of $\mathbf{G}_{\text {ALK }}$ during the CuAAC step ( $\lambda$ ex $546 \mathrm{~nm} / \lambda_{\mathrm{em}} 565 \mathrm{~nm}$, red channel); the third channel corresponds to preexisting lignin, which is detected using its intrinsic autofluorescence at $405 \mathrm{~nm}$ (blue channel). This approach leads to the generation of threecolor localization maps of lignin within plant cell walls that provide precise spatial information on the presence or absence of active lignification machinery between different tissues of an organ (Figure 3A), between different cell types within the same tissue (Figure 3B-C) and within different wall layers of the same cell (Figure 3D). In other words, this methodology allows us to highlight and specifically localize the 'active' lignification sites $\left(\mathbf{H}_{\mathrm{AZ}}\right.$ and $\mathbf{G}_{\mathrm{ALK}}$ channels) by differentiating them from zones where lignin was formed at an earlier stage of plant development (autofluorescence channel). In addition, the sensitivity of the technique is dramatically enhanced when compared to autofluorescence, and much smaller amounts of freshly synthesized lignin can be detected (Figure 3B).

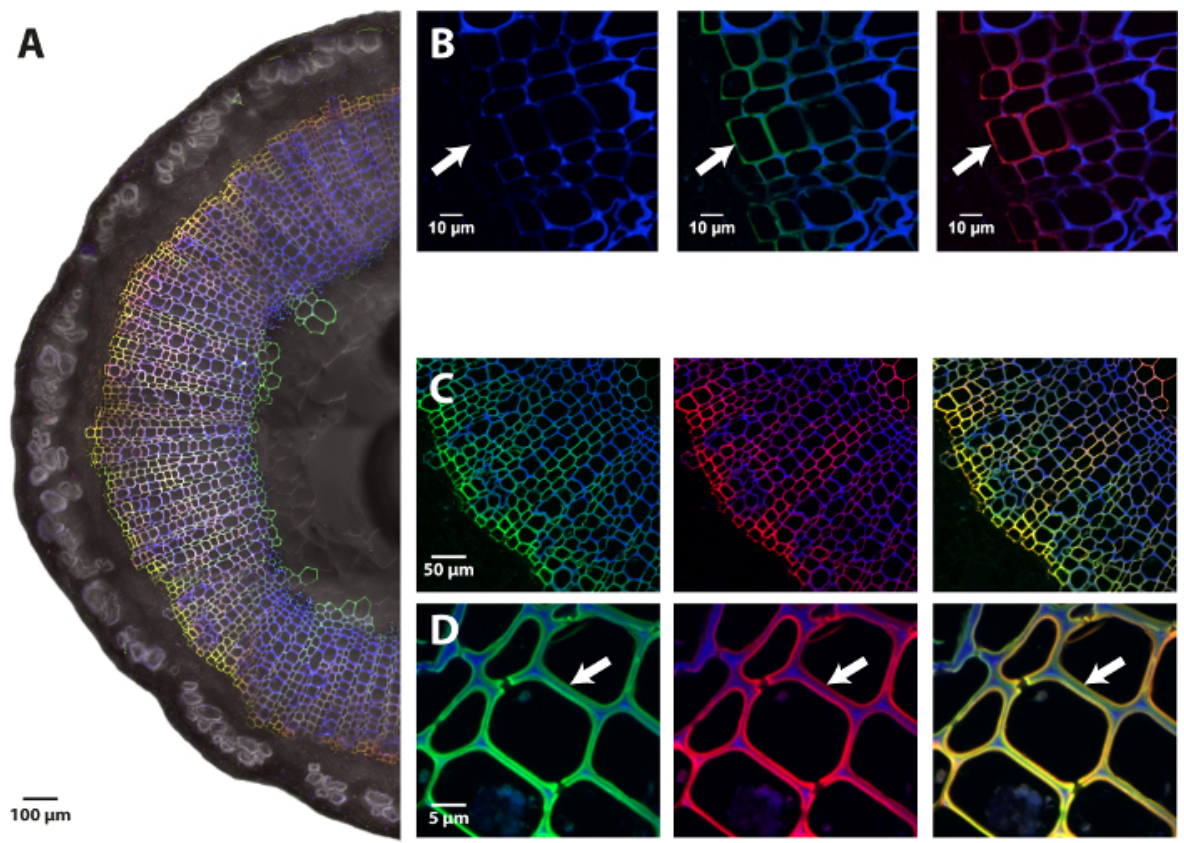

Figure 3: Imaging of incorporated monolignol chemical reporters in Flax Stems. (A) Half of a hand-made transversal section of a flax stem, reconstructed picture. (B) Close-up of the first layers of differentiating xylem. Left panel: lignin autofluorescence (blue, $405 \mathrm{~nm}$ ), middle panel: merged lignin autofluorescence (blue) and $\mathrm{H}_{\mathrm{AZ}}$ fluorescence (green, $526 \mathrm{~nm}$ ) channels, right panel: merged lignin autofluorescence (blue) and $\mathrm{G}_{\mathrm{ALK}}$ fluorescence (red, $565 \mathrm{~nm}$ ) channels. The arrow indicates the first labeled cell wall from the cambium illustrating the increased sensitivity of BLISS compared with autofluorescence. (C) Labeling in different cell types of secondary xylem and (D) close up on secondary xylem cells illustrating the labeling variations in different layers of the same cell wall. Left panel: merged lignin autofluorescence (blue) and $\mathrm{H}_{\mathrm{AZ}}$ fluorescence (green, $526 \mathrm{~nm}$ ) channels, middle panel: merged lignin autofluorescence (blue) and $\mathrm{G}_{\mathrm{ALK}}$ fluorescence (red, $565 \mathrm{~nm}$ ) channels, right panel: merged lignin autofluorescence (blue), $\mathrm{H}_{\mathrm{AZ}}$ (green) and $\mathrm{G}_{\mathrm{ALK}}$ (red) fluorescence channels. $\mathrm{H}_{\mathrm{AZ}}$ and $\mathrm{G}_{\mathrm{ALK}}$ co-localization is depicted in yellow. Please click here to view a larger version of this figure.

Chemical reporter strategies such as the BLISS method presented here or the single-labeling procedures previously published by Tobimatsu et al. ${ }^{8}$ can therefore allow a much finer study than previously accessible methods such as immuno- or histochemical staining while being very simple to implement. For instance, Figure 4 illustrates that the signal intensity for $\mathbf{H}_{\mathrm{AZ}} / \mathbf{G}_{\mathrm{ALK}}$ incorporation in fiber tracheids/vessels (FT) of the flax secondary xylem is very high in the first few cell walls from the cambium but progressively decreases in older cells. This profile is opposite to that of the autofluorescence and indicates that maximum lignification is very rapidly reached in the first two to three FT cell layers from the cambium. In contrast, ray (R) cells appear to continue lignification to much later stages of their development as $\mathbf{H}_{A Z} / \mathbf{G}_{A L K}$ incorporation is much more constant in the walls of all cells from the cambium to the pith. It is not surprising that FT and R cell types display contrasted lignification dynamics, since these cell types have different biological roles with associated differences in their developmental program. FTs are indeed elongated tubes that mature and die rapidly, leaving dead empty cells with thick lignified walls that play essential roles in both mechanical support and vertical transport of water and minerals, whereas the narrow rows of $\mathrm{R}$ cells that compose the xylem rays are alive in their mature and functional state without having thick lignified walls. 

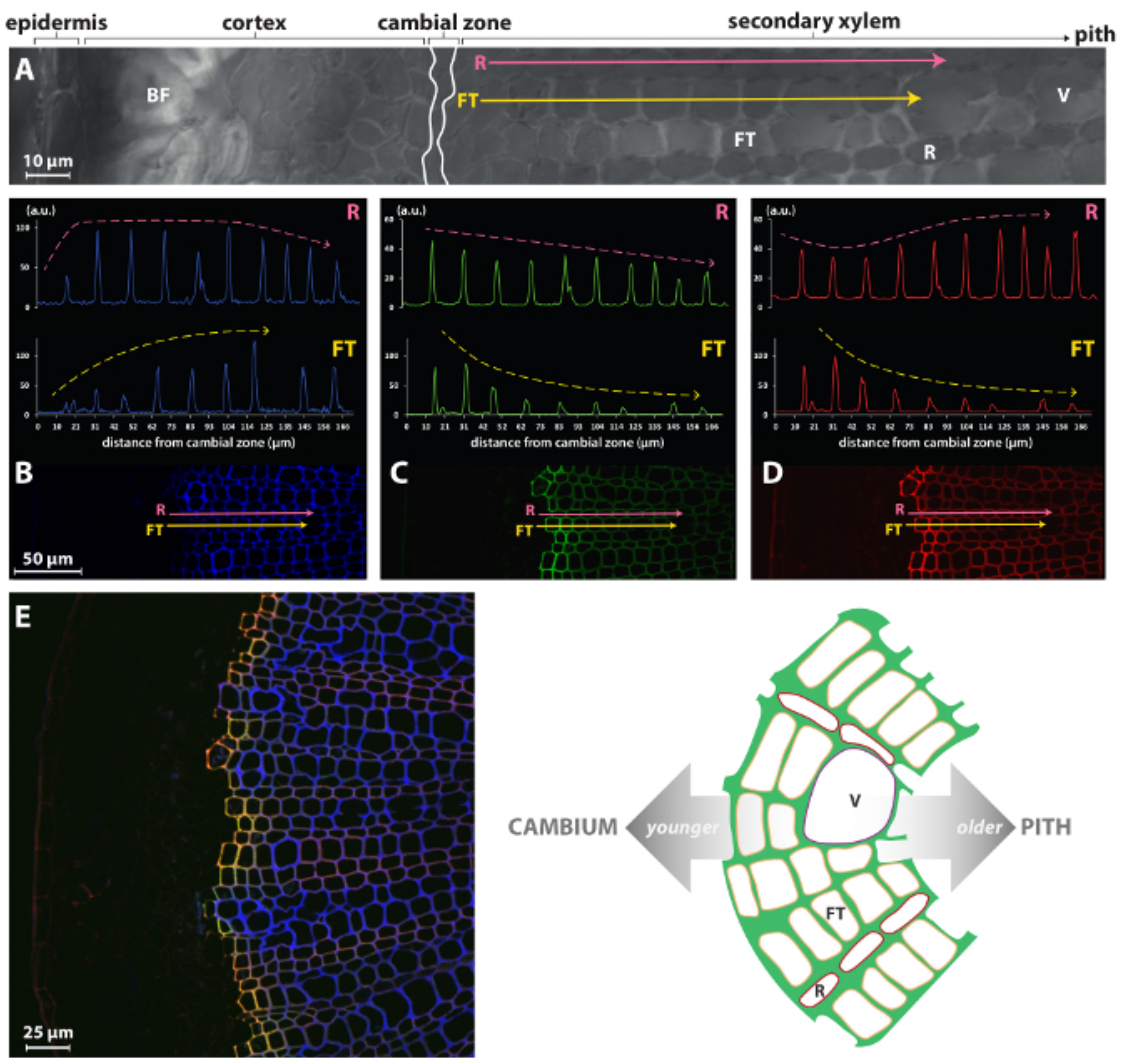

Figure 4: Cell-specific monolignol reporter incorporation in flax xylem. Bright-field confocal microscopy view (A) of part of a freehand crosssection from a flax stem. Pink (ray parenchyma cells, R) and yellow (fiber tracheid cells, FT) arrows indicate vectors spanning from the cambial zone toward the pith and scanned for lignin autofluorescence at $405 \mathrm{~nm}(B), \mathrm{H}_{\mathrm{AZ}}$ fluorescence at $526 \mathrm{~nm}(\mathbf{C})$ and $\mathrm{G}_{A L K}$ fluorescence at $565 \mathrm{~nm}$ (D). (E) View of the secondary xylem. Left panel: merged lignin autofluorescence (blue), $\mathrm{H}_{\mathrm{AZ}}$ (green), and $\mathrm{G}_{\mathrm{ALK}}$ (red) fluorescence channels. $\mathrm{H}_{\mathrm{AZ}}$ and $\mathrm{G}_{\mathrm{ALK}}$ co-localization is depicted in yellow. Right panel: schematic illustration of the secondary xylem structure in the flax stem. V, vessel; $\mathrm{FT}$, fiber tracheid; R, ray parenchyma cell. This figure has been modified from Lion et al. ${ }^{23}$ Please click here to view a larger version of this figure.

In addition, the use of two distinct chemical reporters corresponding to $\mathrm{H}$ - and $\mathrm{G}$-units with two bioorthogonal reactions in the BLISS protocol allows more quantitative results to be obtained. Multiplexed labeling strategies such as BLISS could provide additional insights on the control of monolignol incorporation ratios in various conditions and could contribute to deciphering the elusive lignification process. If the relative percentages of $\mathrm{H}, \mathrm{G}$ and S monolignols in lignins are indeed very variable according to the species, tissue, age or environmental conditions of the plant, the intricate mechanisms that regulate this composition are still not completely understood. The dual labeling technology represents a powerful way of investigating some of the parameters that regulate lignin composition. For example, varying the $\mathbf{H}_{A Z}: \mathbf{G}_{A L K}$ ratio with $B L I S S$ allowed us to demonstrate that lignin composition in secondary xylem tissues is directly dependent upon monolignol availability within the cell walls, rather than on peroxidase/laccase specificity (Figure 5). 
${ }^{*} \mathrm{H}_{N 2}:{ }^{*} \mathrm{G}_{\mathrm{ALK}}$

$90: 10$

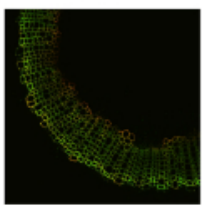

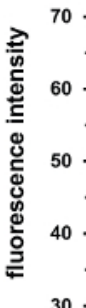

1
$80: 20$
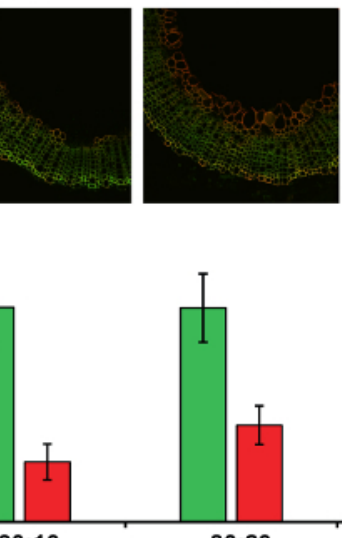

$0: 10$
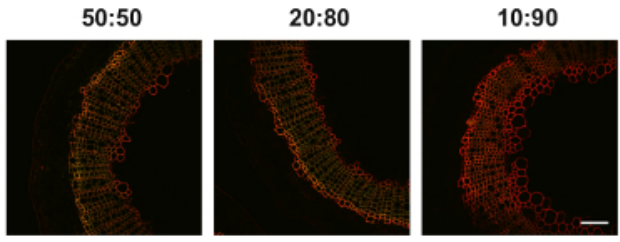

Figure 5: Effect of Incubating Flax Stem Sections with Different Percentage Ratios of $\mathbf{H}_{A Z}$ and $\mathbf{G}_{A L K} . \mathrm{H}_{A Z}: \mathrm{G}_{A L K} \%$ ratios are given above each column of figures. Top: merged $H_{A Z}$ and $G_{A L K}$ channels. Bottom: histograms indicating average fluorescence intensity of $H_{A Z}$ and $G_{A L K}$ for the different\% ratios. Values are expressed as mean of the mean fluorescence intensity in gray levels \pm SD. Scale bar $=100 \mu \mathrm{m}$. This figure has been modified from Lion et al. ${ }^{23}$ Please click here to view a larger version of this figure.

Flax is grown for its bast fibers (BF) that are used to manufacture textiles, luxury papers or environmentally-friendly composite materials. An important aspect of their industrial valorization is that they contain very low levels of lignin in their cell walls, which are characterized by an extremely thick S2/G secondary layer ${ }^{19,24}$. BLISS can highlight lignification dynamics differences between the different layers of the same cell wall. Figure $6 \mathrm{~A}$ shows that $\mathbf{H}_{\mathrm{AZ}}$ and $\mathbf{G}_{\mathrm{ALK}}$ reporter incorporation are limited to the cell corners and middle lamella/primary cell wall of some but not all bast fibers. The total absence of lignification in the thick bast fiber secondary cell wall layer even when monolignol chemical reporters are exogenously supplied reveals that their hypolignified state arises from the absence of a molecular environment suitable for enzymaticallymediated oxidation and incorporation of monolignols into a growing polymer chain, and that it is not just due to transcriptional regulation of monolignol biosynthesis genes as previously reported ${ }^{25}$. This observation also correlates well with the fact that flax peroxidase genes are upregulated in the outer stem tissues of the flax chemical Ibf1 mutant that possesses lignified bast fibers ${ }^{26}$.
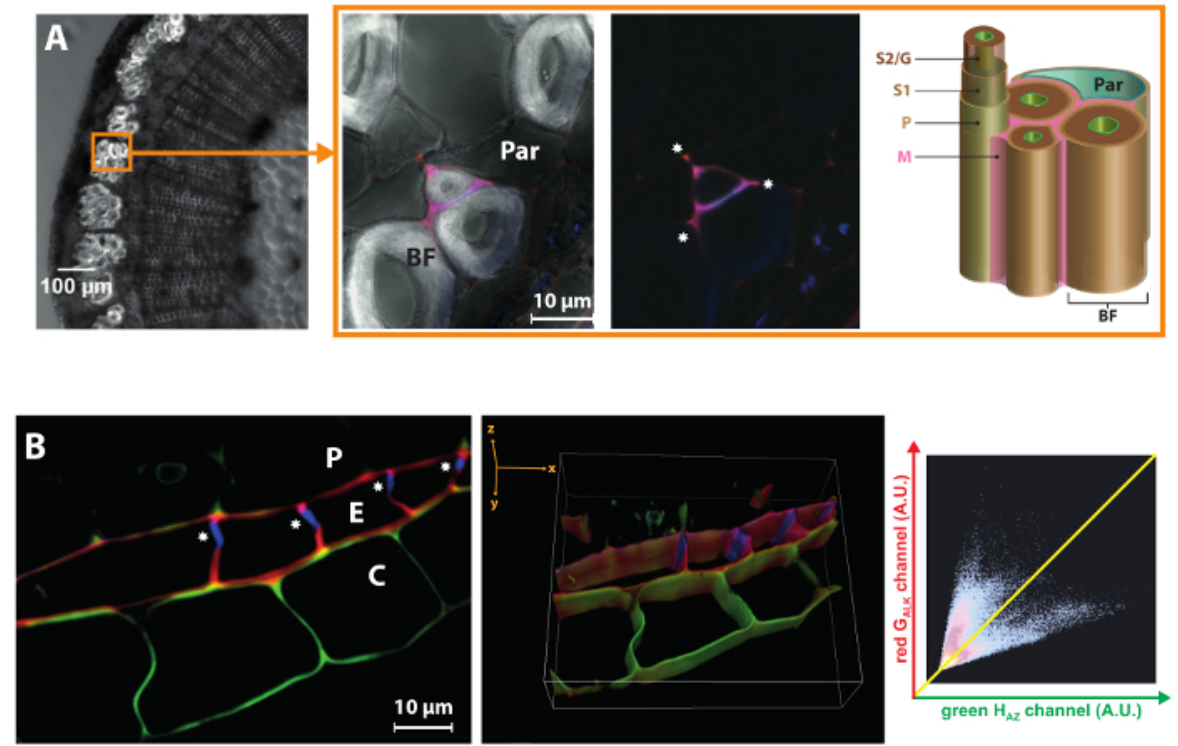

Figure 6: BLISS highlights cell wall substructure- or layer-specific differences. (A) Left panel: bright-field image of a flax stem section. The circle indicates a fiber bundle. Right panel: close up on bast fibers. Merged $\mathrm{H}_{\mathrm{AZ}}$ and $\mathrm{G}_{\mathrm{ALK}}$ channels (with and without bright-field) showing that lignification is limited to cell corners $(\star)$ and middle lamella/primary cell wall of some bast fibers. BF, bast fiber; Par, parenchyma cell; M, middle lamella; P, primary cell wall; S1, first layer of secondary cell wall; S2/G, secondary layer/gelatinous layer of secondary cell wall. (B) 2D slice and $3 \mathrm{D}$ reconstruction of confocal z-stack zoom of flax root endodermis region. The Casparian strip ( $\star$ ) only displays autofluorescence and does not incorporate $\mathrm{H}_{A Z}$ or $\mathrm{G}_{\mathrm{ALK}}$. The associated fluorogram shows a $\mathrm{G}_{\mathrm{ALK}} / \mathrm{H}_{\mathrm{AZ}}$ anti-correlation: high green fluorescence is associated to low red signal (cortex) and vice-versa (endodermis). Pericycle (P), Endodermis (E), Cortex (C). Please click here to view a larger version of this figure. 
Finally, this two-color methodology can also provide valuable information on the 'lignification state' of cell wall substructures at various developmental stages and in plant organs other than the stem. For example, the endodermis of flax roots is characterized by the existence of a Casparian band in its radial and transverse cell walls during the early stages of development. This band of hydrophobic biopolymer, made of suberin and/or lignin, prevents water and solutes taken up by the root from entering passively through the apoplast and forces them to pass through the plasma membrane via a symplastic route thereby contributing to the selective uptake capacities of plant roots. When applied to flax roots, our strategy showed that $\mathrm{H}_{A Z}$ and $\mathrm{G}_{\mathrm{ALK}}$ are incorporated in the tangential walls of endodermal cells as well as in parts of the radial walls where the Casparian band is absent (Figure 6B). However, total absence of monolignol reporter incorporation in the Casparian band itself indicates that it is mature at this developmental stage while the other walls are still the site of further biopolymer deposition. The reconstructed $3 \mathrm{D}$ view of a z-stack clearly brings the Casparian band to light. Interestingly, the walls of some cortical cells adjacent to the endodermis also proved capable of incorporating $\mathrm{H}_{\mathrm{AZ}}$ preferentially (thereby indicating the presence of lignin/suberin in the flax root cortex) but not $\mathrm{G}_{\mathrm{ALK}}$. Colocalization analysis showed an anti-correlation between $H_{A Z}$ and $G_{A L K}$, which suggest the existence of cell-specific wall structure/enzymatic machinery in these two adjacent cell types.

Supplemental Table 1: Preparation of solid 1/2 MS Medium Please click here to download this file.

Supplemental Table 2: Preparation of chemical reporter stock solutions Please click here to download this file.

\section{Discussion}

As previously mentioned, the dual labeling BLISS protocol presented in this paper is one of the first examples of a SPAAC/CuAAC combination in vivo ${ }^{12,23}$. Each step was thoroughly optimized and validated, and it is very important that the order in which the two click chemistry labeling reactions are sequentially performed is respected (i.e., SPAAC first, followed by CuAAC). All cross-controls showed that each labeling step is specific when the BLISS protocol is applied ${ }^{23}$ : carrying out the SPAAC step first leads to the highly chemoselective labeling of $\mathbf{H}_{A Z}$ azide functions by the cyclooctyne-functionalized fluorophore through a [3+2] cycloaddition reaction with fast kinetics. Once $\mathbf{H}_{\mathrm{AZ}}$ units are tagged, the CUAAC step necessitating copper(I) catalyzed activation of $\mathbf{G}_{\mathrm{ALK}}$ terminal alkynes to generate triazole links by reaction with the azide-fluor 545 probe can be carried out. In contrast, the reverse order (i.e., CuAAC first, followed by SPAAC) should not be used as it leads to $\mathbf{G}_{\mathrm{ALK}}$ and $\mathbf{H}_{\mathrm{AZ}}$ unit cross-coupling, which competes with fluorophore ligation and induces a dramatic loss of signal. It is also important to stress the necessity of the intermediate washing steps to avoid non-specific staining.

We have shown that our method can be applied to various biological experiment designs. The BLISS labeling protocol was first applied to freehand cross-sections of flax stems (approximately 150-250 $\mu \mathrm{m}$ thick) that were previously cut and incubated with the click-ready monolignols. Although this design has the advantage of minimizing the needed quantities of chemical reporter (as incubation volumes are reduced) and of facilitating the production of statistical replicates, it is not, strictly speaking, an in vivo system and in some cases, may not reflect all aspects of real spatio-temporal lignification dynamics. In a second experimental design, we therefore adapted the BLISS protocol to a method that was previously used to study the incorporation of radiolabeled monolignols in pine and gingko ${ }^{27}$. In this approach, the roots and stem of the plant are physically separated and the base of the whole stem is incubated in the monolignol solution in what may be dubbed the 'flower vase' approach. After leaving the stems the desired (incubation) time, cross-sections are cut and the BLISS protocol performed. This allowed us to show (i) that the modified monolignols are transported through the living stem and are incorporated in growing lignin polymers within the cell walls and (ii) that the localization pattern was essentially identical to that of the cross-section approach. This type of experiment has the merit of being performed in a real living plant / live cell approach allowing longer experiments and more in-depth studies, but requires greater quantities of chemical reporter. Finally, the BLISS protocol was also used with flax plant seedlings, representing a real living plant model in which the chemical reporters must be absorbed through the roots before being transported up the stem. While this model has the clear advantage of being performed in living plants, in practice, it is limited to young seedlings and is not really suitable for investigating lignification dynamics in older plants for practical reasons (long incubation time, elevated quantity of chemical reporters). Nevertheless, these three experiment designs are complementary and all have their pros and cons with regards to practical aspects and biological significance depending on the type of biological question to be answered.

Developed to study the lignification dynamics in flax, our protocol is highly adaptable, not only in terms of biological experiment design but also in terms of its application to other plant species and organs/tissues. For instance, BLISS can easily be transferred to the Arabidopsis or the Populus genera that are more amenable to studies with knock-out or knock-down mutants for various genes. In principle, dual labeling studies with our approach can also be extended to other biomolecules by using two distinct modified precursors of plant cell wall polymers - including all three main monolignols or their metabolic precursors as well as various monosaccharides that constitute the polysaccharide matrix. Since its inception, bioorthogonal chemistry has indeed been mainly developed to investigate glycans/polysaccharides through metabolic oligosaccharide engineering (MOE) $)^{4,5,17,28}$, but surprisingly there have been only very few applications to plant biology so far ${ }^{7,8,9,10,11,12}$. In terms of compatibility of the reactions, the study of lignin was indeed a complex case to solve as both chemical reporters are incorporated into the same reticulated polymer. The possibility of unlabeled $\mathbf{H}_{A Z}-\mathbf{G}_{A L K}$ cross-link formation was the major issue to overcome due to the spatial proximity of $\mathbf{G}_{A L K}$ and $\mathbf{H}_{A Z}$ units within the $3 \mathrm{D}$ structure of lignin ${ }^{23}$, a limitation that may not be present if the two chemical reporters are not incorporated in the same type of biopolymer or in the same spatial region of any given cell.

In a wider scope the BLISS methodology could essentially be applied to any two-color fluorescence imaging study in bacterial or animal models using two distinct chemical reporters bearing an azide and terminal alkyne tag, respectively.

\section{Disclosures}

The authors declare that they have no competing financial interests. 


\section{Acknowledgements}

We are indebted to the Research Federation FRABio and the TisBio imaging platform (Univ. Lille, CNRS, FR 3688, FRABio, BiochimieStructurale et Fonctionnelle des Assemblages Biomoléculaires) for providing the technical environment conducive to achieving this work.

\section{References}

1. Grammel, M., \& Hang, H. C. Chemical reporters for biological discovery. Nat Chem Biol. 9 (8), $475-484$ (2013).

2. Prescher, J. A., \& Bertozzi, C. R. Chemistry in living systems. Nat Chem Biol. 1 (1), 13-21 (2005).

3. Sletten, E. M., \& Bertozzi, C. R. Bioorthogonal chemistry: fishing for selectivity in a sea of functionality. Ange Chemie (Int Ed. Engl). 48 (38), 6974-6998 (2009).

4. Chang, P. V. et al. Metabolic labeling of sialic acids in living animals with alkynyl sugars. Ange Chemie (Int Ed. Engl). 48 (22), $4030-4033$ (2009).

5. Gilormini, P. A. et al. A sequential bioorthogonal dual strategy: ManNAl and SiaNAl as distinct tools to unravel sialic acid metabolic pathways. Chem. Commun. 52 (11), 2318-2321 (2016).

6. Mbua, N. E. et al. Abnormal accumulation and recycling of glycoproteins visualized in Niemann-Pick type C cells using the chemical reporter strategy. Proc Natl Acad Sci U S A . 110 (25), 10207-10212 (2013).

7. Anderson, C. T., Wallace, I. S., \& Somerville, C. R. Metabolic click-labeling with a fucose analog reveals pectin delivery, architecture, and dynamics in Arabidopsis cell walls. Proc Natl Acad Sci U S A . 109 (4), 1329-1334 (2012).

8. Tobimatsu, Y. et al. A click chemistry strategy for visualization of plant cell wall lignification. Chem. Commun. 50 (82), 12262-12265 (2014).

9. Bukowski, N. et al. Development of a clickable designer monolignol for interrogation of lignification in plant cell walls. Bioconjugate Chem. $\mathbf{2 5}$ (12), 2189-2196 (2014).

10. Pandey, J. L. et al. A versatile click-compatible monolignol probe to study lignin deposition in plant cell walls. PLOS ONE. 10 (4), e0121334 (2015).

11. Pandey, J. L. et al. Investigating biochemical and developmental dependencies of lignification with a click-compatible monolignol analog in Arabidopsis thaliana stems. Front Plant Sci. 7 (2016).

12. Zhu, Y., Wu, J., \& Chen, X. Metabolic labeling and imaging of $N$-linked glycans in Arabidopsis thaliana. Ange Chemie (Int Ed. Engl). 55 (32), 9301-9305 (2016).

13. Boerjan, W., Ralph, J., \& Baucher, M. Lignin biosynthesis. Annu Rev Plant Biol . 54, 519-546 (2003).

14. Weng, J.-K., \& Chapple, C. The origin and evolution of lignin biosynthesis. New Phytol. 187 (2), 273-285 (2010).

15. Mottiar, Y., Vanholme, R., Boerjan, W., Ralph, J., \& Mansfield, S. D. Designer lignins: harnessing the plasticity of lignification. Curr Opin Biotechnol. 37, 190-200 (2016).

16. Rinaldi, R. et al. Paving the way for lignin valorisation: recent advances in bioengineering, biorefining and catalysis. Ange Chemie (Int Ed.). 55 (29), 8164-8215 (2016).

17. Wratil, P. R., Horstkorte, R., \& Reutter, W. Metabolic glycoengineering with $N$-acyl side chain modified mannosamines. Ange Chemie (Int Ed. Engl). 55 (33), 9482-9512 (2016).

18. Feng, L. et al. Bifunctional unnatural sialic acids for dual metabolic labeling of cell-surface sialylated glycans. J Am Chem Soc. 135 (25), 9244-9247 (2013).

19. Dumont, M. et al. Plant cell wall imaging by metabolic click-mediated labelling of rhamnogalacturonan II using azido 3-deoxy- $d$ - manno oct-2-ulosonic acid. Plant J. 85 (3), 437-447 (2016).

20. Niederwieser, A. et al. Two-color glycan labeling of live cells by a combination of Diels-Alder and click chemistry. Ange Chemie Int Ed. $\mathbf{5 2}$ (15), 4265-4268 (2013).

21. Späte, A.-K. et al. Exploring the potential of norbornene-modified mannosamine derivatives for metabolic glycoengineering. Chem Bio Chem. 17 (14), 1374-1383 (2016).

22. Späte, A.-K. et al. Rapid Labeling of metabolically engineered cell-surface glycoconjugates with a carbamate-linked cyclopropene reporter. Bioconjugate Chem. 25 (1), 147-154 (2014).

23. Lion, C. et al. BLISS: a bioorthogonal dual-labeling strategy to unravel lignification dynamics in plants. Cell Chem Biol. 24 (3), $326-338$ (2017).

24. del Río, J. C. et al. Structural characterization of guaiacyl-rich lignins in flax (Linum usitatissimum) fibers and shives. J Agric Food Chemist. 59 (20), 11088-11099 (2011).

25. Huis, R. et al. Natural hypolignification is associated with extensive oligolignol accumulation in flax stems. Plant Physiol. 158 (4), $1893-1915$ (2012).

26. Chantreau, M. et al. Ectopic lignification in the flax lignified bast fiber1 mutant stem is associated with tissue-specific modifications in gene expression and cell wall composition. The Plant Cell. 26 (11), 4462-4482 (2014).

27. Terashima, N., Ko, C., Matsushita, Y., \& Westermark, U. Monolignol glucosides as intermediate compounds in lignin biosynthesis. Revisiting the cell wall lignification and new ${ }^{13} \mathrm{C}$-tracer experiments with Ginkgo biloba and Magnolia liliiflora. Holzforschung. 70 (9), $801-810$ (2016).

28. Noel, M. et al. Probing the CMP-sialic acid donor specificity of two human $\beta$-d-galactoside sialyltransferases (ST3Gal I and ST6Gal I) selectively acting on O- and N-glycosylproteins. Chembiochem: Eur J Chem Biol. (2017). 